\title{
Renal Duplex Doppler Ultrasonography: A Noninvasive Predictor of Kidney Dysfunction and Hepatorenal Failure in Liver Disease
}

\author{
Joel F. Platt, ${ }^{1}$ James H. Ellis, ${ }^{1}$ Jonathan M. Rubin, ${ }^{1}$ Robert M. Merion ${ }^{2}$ AND Michael R. LuCEy, ${ }^{3}$ \\ ${ }^{1}$ Department of Radiology, ${ }^{2}$ Department of Surgery and ${ }^{3}$ Department of Internal Medicine, University of Michigan Medical \\ Center, Ann Arbor, Michigan 48109-0030
}

\begin{abstract}
Hepatorenal failure, a well-recognized complication of established liver disease, is characterized by early renal hemodynamic changes (vasoconstriction) before clinically recognized kidney disease. This renal vasoconstriction (increased renal vascular resistance) should be detectable noninvasively by Doppler ultrasonography. We studied whether renal Doppler ultrasonography detects abnormalities in patients with nonazotemic liver disease and its prognostic value for subsequent kidney status. We observed by renal Doppler ultrasonography 180 patients who had liver disease without azotemia. A simple parameter, resistive index, was derived for each subject on the basis of Doppler waveform analysis. Traditional parameters used to assess patients with liver disease were also recorded at the time of Doppler ultrasonography. Subsequent kidney outcomes were kidney dysfunction (doubling of initial creatinine level to $1.5 \mathrm{mg} / \mathrm{dl}[133$ $\mu \mathrm{mol} / \mathrm{L}])$ or more and the presence or absence of the hepatorenal syndrome. Abnormal results of Doppler examinations (elevated resistive index) were seen in 76 (42\%) of the 180 patients. Kidney dysfunction developed in $55 \%(42 / 76)$ of the patients with an elevated resistive index and $6 \%(6 / 104)$ of those with normal results of Doppler study $(p<0.00005)$. Hepatorenal syndrome developed in $26 \%(20 / 76)$ of subjects with an elevated resistive index and $1 \%(1 / 104)$ of those with a normal resistive index $(p<0.00005)$. Cox regression analysis identified resistive index as a significant independent predictor of subsequent hepatorenal syndrome $(p<0.00005)$ and kidney dysfunction $(p<$ 0.00005). Renal duplex Doppler ultrasonography can noninvasively identify a subgroup of nonazotemic patients with liver disease that is at significantly higher risk for subsequent development of kidney dysfunction and the hepatorenal syndrome. (HEPATOLOGY 1994;20:362-369.)
\end{abstract}

Kidney dysfunction commonly develops in patients

Received August 16, 1993; accepted March 4, 1994.

Address reprint requests to: Joel F. Platt, M.D., Department of Radiology, University of Michigan Medical Center, 1500 E. Medical Center Drive, Ann Arbor, MI 48109-0030.

Copyright 1994 by the American Association for the Study of Liver Diseases

$0270-9139 / 94 \$ 3.00+0 \quad 31 / 1 / 56428$ with established liver disease (1-3). In its most severe form this kidney dysfunction is termed the hepatorenal syndrome, which has been defined as unexplained kidney failure in a patient with liver disease who does not have clinical, laboratory or anatomic evidence of other known causes of kidney failure (1). The progressive kidney dysfunction that accompanies liver disease is generally considered to be functional in nature because consistent pathologic changes are absent, because the kidney failure can be reversed with timely liver transplantation, and because kidneys in patients with the hepatorenal syndrome can be successfully transplanted into patients with normal livers (4-6).

Renal hemodynamic changes begin early in the course of liver disease-related functional kidney failure, even before changes in serum creatinine concentration are detectable $(2,7-10)$. The hallmark change is intense intrarenal vasoconstriction $(1,8,9)$. This vasoconstriction is associated with a reduced renal plasma flow and an elevated renal arterial vascular resistance that may precede clinically recognized kidney dysfunction by weeks or months. Although the precise cause of the renal vasoconstriction remains elusive and is likely multifactorial (11), a state of elevated renal vascular resistance is present in many nonazotemic patients with liver disease. These patients may be at greater risk for subsequent development of overt hepatorenal syndrome $(2,7)$.

Duplex Doppler ultrasonography is a widely used noninvasive method to assess vascular patency and blood flow in many sites. Duplex Doppler can be used to assess vascular resistance in the small renal intraparenchymal vessels through simple analysis of the Doppler waveform by a parameter termed the resistive index (RI) (12). An elevated RI (reflecting intrarenal vasoconstriction) has been observed in various conditions associated with elevated renal vascular resistance such as kidney obstruction (13), acute tubular necrosis (14), renal vein thrombosis (15) and the hemolytic-uremic syndrome (16) and should be detectable in liver diseaserelated functional kidney failure.

In a previous pilot study in patients before and after liver transplantation we found Doppler analysis helpful in predicting subsequent kidney status and to some 


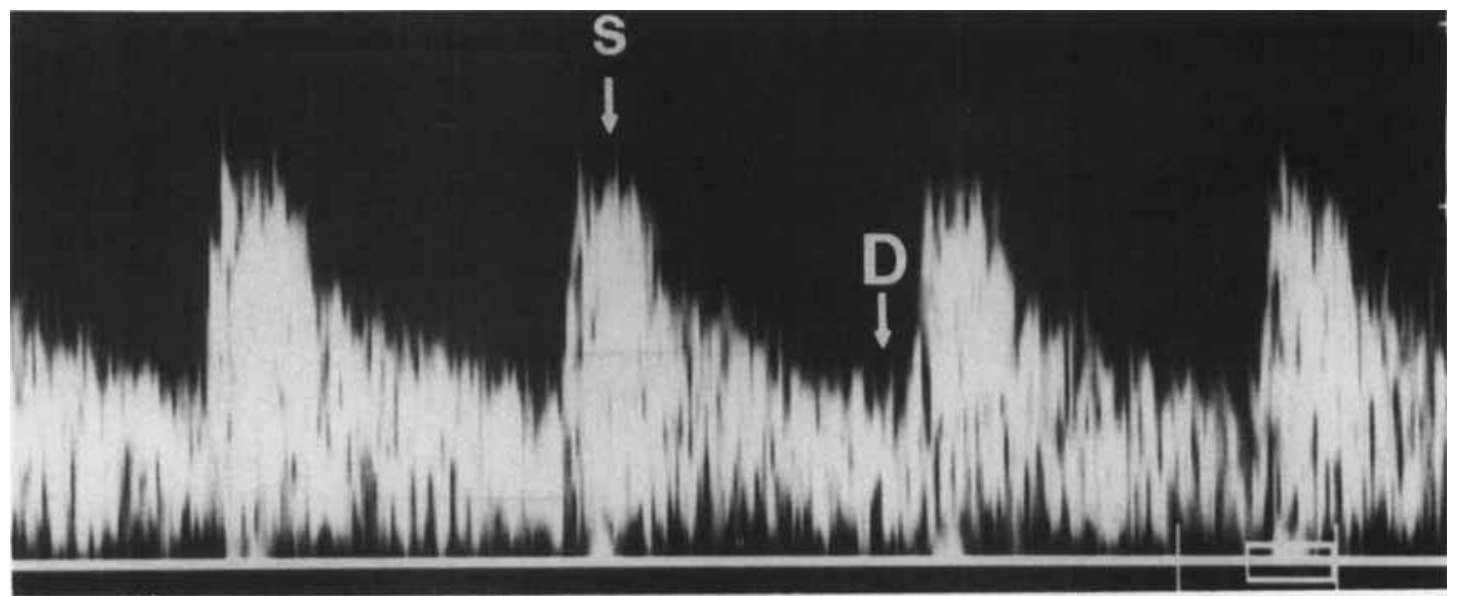

FIG. 1. Normal intrarenal Doppler waveform showing determination of RI. $S$, Peak systole; $D$, minimum diastole. RI $=(\mathrm{S}-\mathrm{D}) / \mathrm{S}$.

extent in predicting posttransplantation morbidity (17). Another recent study found elevated RIs in more than half of nonazotemic patients with significant cirrhosis but did not follow the patients to assess the chance of the development of overt kidney dysfunction (18). The goal of the current study was to assess the prognostic value of duplex Doppler ultrasonography in a large population of nonazotemic patients with liver disease. Early identification of those patients with established liver disease who are at particular risk for the development of the hepatorenal syndrome may be beneficial both because clinical management may be modified to avoid other nephrotoxins (drugs, radiographic contrast) and because overt kidney failure has been identified as an independent risk factor for outcome in liver transplantation (19).

\section{PATIENTS AND METHODS}

Patients were included in this study population if they had been evaluated for known liver disease at our institution's liver clinic and did not have clinical azotemia (serum creatinine concentration $\leq 1.5 \mathrm{mg} / \mathrm{dl}[133 \mu \mathrm{mol} / \mathrm{L}]$ ) and if a minimum period of 4 mo of follow-up clinical and laboratory data was available. Three hundred seven patients were initially prospectively observed in our liver clinics over a 20 -mo period. Of these, 67 were excluded because their serum creatinine concentration at presentation exceeded $1.5 \mathrm{mg} / \mathrm{dl}$. An additional 60 nonazotemic patients were excluded because no follow-up clinical and laboratory data were available for a minimum of 4 mo to assess subsequent kidney status.

The remaining 180 patients constitute our study population. All of these patients with liver disease had a serum creatinine concentration of $1.5 \mathrm{mg} / \mathrm{dl}(133 \mu \mathrm{mol} / \mathrm{L})$ or less at the time of duplex Doppler ultrasonography and a minimum of 4 mo clinical follow-up unless hepatorenal syndrome developed before this minimum follow-up time. The clinical characteristics of the study population are shown in Table 1.

Multiple clinical and laboratory parameters were assessed at the time of the duplex Doppler ultrasound examination. The patient's systolic and diastolic blood pressures were recorded, allowing for calculation of mean arterial pressure [Diastolic pressure $+1 / 3$ (Systolic - diastolic)]. Laboratory values included serum albumin (level grams per deciliter), serum bilirubin level (milligrams per deciliter), prothrombin time (PT, seconds), serum sodium concentration (millimoles per liter) and serum creatinine concentration (milligrams per deciliter). Ascites was assessed by sonography and graded as absent, mild or moderate to severe. Sixty-three patients had no ascites, and the remaining 117 patients did have ascites. Encephalopathy was clinically assessed and classified as absent, grade 1 to 2 encephalopathy or grade 3 to 4 encephalopathy. Patient age was also recorded in years at time of Doppler ultrasound study.

In addition to considering the above parameters individually, we combined five factors (serum albumin, serum bilirubin, ascites, encephalopathy and PT) to grade the severity of disease on the basis of the Pugh modification of the Child-Turcotte classification (20).

For each patient the zero time was defined as the day of the duplex Doppler ultrasound study. The number of days of follow-up in each patient was recorded. End points for follow-up were, where applicable, death, hepatorenal syndrome, day of liver transplantation (no data on kidney status after liver transplantation were included in this study) and last day of normal follow-up. One hundred sixteen patients in all underwent liver transplantation during the study period. If kidney status remained stable, a minimum of 4 mo follow-up was required for inclusion in the study. The two kidney status outcome changes identified were kidney dysfunction and hepatorenal syndrome. We defined kidney dysfunction as a doubling of the initial creatinine level (recorded at time of Doppler ultrasonography) to a level of $1.5 \mathrm{mg} / \mathrm{dl}(133 \mu \mathrm{mol} / \mathrm{L})$ or greater. We used this outcome parameter to identify all patients in whom significant kidney disease developed during the follow-up period. Thus the kidney dysfunction group includes, in addition to the patients with overt hepatorenal syndrome, those patients in whom kidney deterioration developed that was not definitely categorized as representing hepatorenal syndrome. We defined hepatorenal syndrome clinically on the basis of usual criteria: kidney failure developing without apparent cause in a patient with liver disease, urine sodium level more than $20 \mathrm{mmol} / \mathrm{L}$, benign urinary sediment, oliguria ( $<400 \mathrm{ml} /$ day) and lack of response to fluid challenge as proved by central venous monitoring $(1,3,11)$.

All patients had a prospectively obtained Doppler ultrasound study with the results blinded to those managing the clinical follow-up in these patients. The duplex Doppler ultrasonography technique is summarized as follows (12): in 


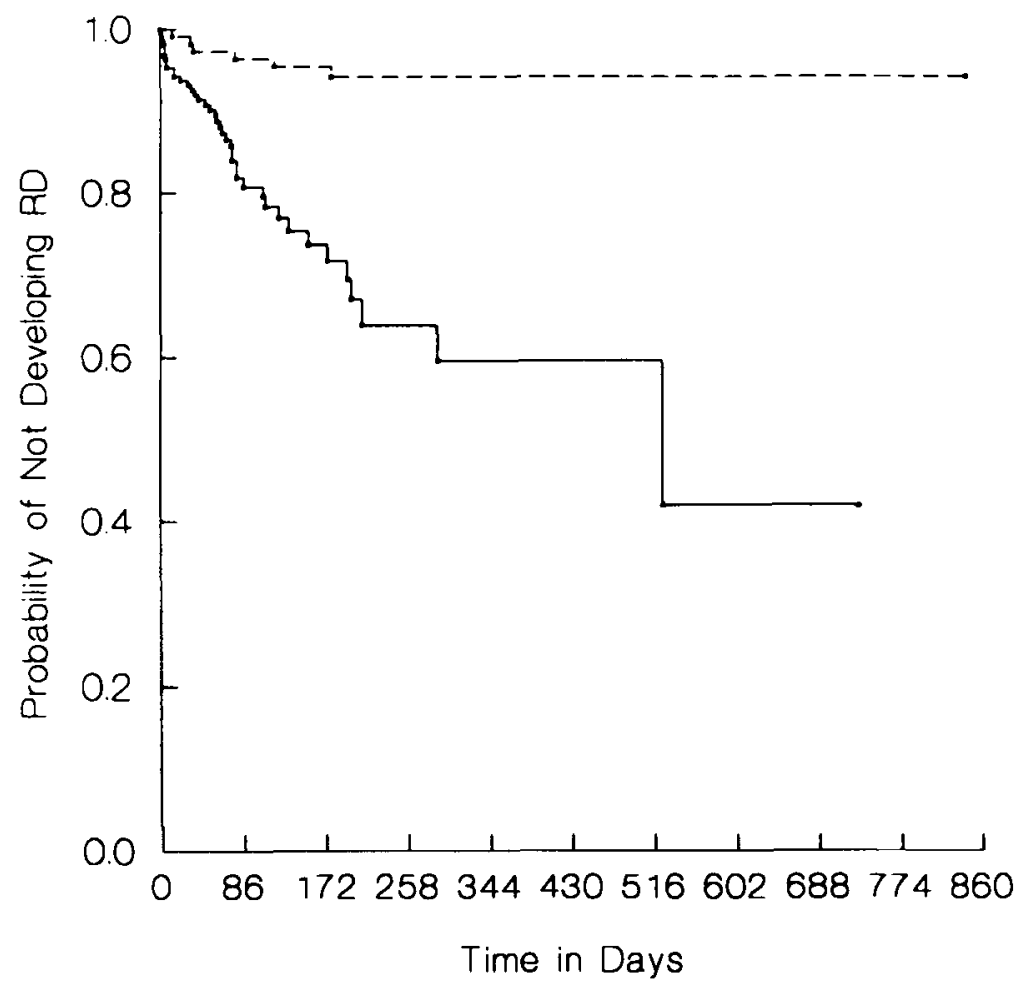

FIG. 2. Survival-type curves demonstrating probability of kidney dysfunction $(R D)$ developing or not developing over time. Dashed line, Patients with normal renal RI; solid line, patients with abnormal renal RI. Difference between curves is statistically significant ( $p<0.00005$ ). Number of patients in follow-up group was 128 at 172 days, 106 at 258 days, 81 at 344 days and 31 at 430 days.

each kidney, real-time ultrasound examinations were performed with a $3.5-\mathrm{MHz}$ transducer (model 128; Acuson, Mountain View, CA) or a 3-MHz transducer (model UM 8 or 9; Advanced Technology Laboratories, Bothell, WA), and a pulsed Doppler evaluation of intrarenal arteries was performed. Multiple Doppler signal tracings and a standard gray-scale examination of the kidney were recorded on film. Doppler signals were, in general, obtained from arcuate arteries at the corticomedullary junction, interlobar arteries along the border of medullary pyramids or both. The Doppler waveforms were made on the lowest frequency-shift range possible without aliasing. This procedure maximized the size of the Doppler spectrum and decreased the percentage error in the measurements. In addition, we used the lowest possible wall filter for each ultrasound scanner. Doppler sample volume was set at 2 to $5 \mathrm{~mm}$.

Duplex Doppler ultrasound examinations required $15 \mathrm{~min}$ or less in most patients. Movement of the patient and inability of the patient to suspend respiration temporarily were the most common causes of a more prolonged, difficult examination. The RI [(Peak systolic frequency shift - Minimum diastolic frequency shift)/Peak systolic frequency shift] was determined by hand measurement of the hard copy with calipers (Fig. 1). The RI for each kidney was calculated as an average value obtained from three to five waveforms recorded in three different regions of the kidney. Subsequently, a mean renal RI was calculated for each patient (average of both kidneys). The person performing the duplex Doppler ultrasound examinations and renal RI measurements was blinded to the patients' clinical status and laboratory results. In addition to the actual $R I$ value being recorded, it was classified as normal or abnormal on the basis of a threshold RI value. On the basis of multiple prior studies $(12,13,16)$ an RI of 0.70 or more was considered abnormal (indicative of elevated renal vascular resistance and renal vasoconstriction). In general, the amount of variation in RI for different sites within a particular kidney was small, averaging $3.5 \%$ in patients with a normal RI and $4.3 \%$ in those with an elevated RI. Although our study did not primarily seek to evaluate longitudinal changes in RI for a given patient, in a small subset of patients $(n=20)$ a second Doppler examination was performed less than 1 wk after the initial study. The mean RI difference for these two studies was $3.2 \%$ with no follow-up study result differing by more than $5 \%$ from the original RI.

Analysis of RI vs. development of kidney dysfunction or hepatorenal syndrome used the $\chi^{2}$ test. To assess the prognostic value of individual variables in our study, we used a Cox proportional hazard regression analysis (BMDP program; BMDP Statistical Software, Inc., Los Angeles, CA). This method was used to determine whether RI had significant predictive value above and beyond the commonly used factors to assess severity and prognosis in patients with liver disease. The covariables chosen were age, serum albumin level, serum bilirubin level, serum sodium level, PT, serum creatinine concentration, mean arterial pressure, ascites grade and encephalopathy grade. Estimated survival-type function curves were derived for RI status controlling for covariants with respect to the outcomes of hepatorenal syndrome and kidney dysfunction. These results were depicted graphically. Differences between the elevated RI and normal RI groups were analyzed with unpaired Student's $t$-test where appropriate.

The duplex Doppler ultrasonography protocol and study were approved by our local institutional review board. Patients 
TABle 1. Characteristics of 180 patients with liver disease

\begin{tabular}{lc} 
Sex & $102(57 \%)$ \\
$\mathrm{M}$ & $78(43 \%)$ \\
$\mathrm{F}$ & \\
Age (yr) & 47 \\
Mean & $8-66$ \\
Range & \\
Principal cause of liver disease & 66 \\
Alcoholic cirrhosis & 44 \\
Cryptogenic cirrhosis & 18 \\
PBC & 13 \\
Primary sclerosing cholangitis & 9 \\
CAH & 30 \\
Miscellaneous & \\
Laboratory values & \\
Creatinine $(\mathrm{mg} / \mathrm{dl})^{b}$ & $0.90 \pm 0.23$ \\
Albumin (gm/dl) & $3.1 \pm 0.6$ \\
PT (sec) & $14.5 \pm 2.1$ \\
Bilirubin (mg/dl) & $4.8 \pm 3.7$ \\
\hline
\end{tabular}

${ }^{\alpha}$ Data expressed as mean \pm 1 S.D.

${ }^{6}$ To convert values for creatinine to micromoles per liter multiply by 88.4 .

'To convert bilirubin to micromoles per liter multiply by 17.1 .

were already undergoing clinically requested hepatic vessel Doppler sonograms and consented to the renal vessel studies.

\section{RESULTS}

Of the 180 patients with established liver disease who fulfilled the study entry criteria including serum creatinine level of $1.5 \mathrm{mg} / \mathrm{dl}$ or less, $76(42 \%)$ had an elevated $\mathrm{RI}$ (indicative of renal vasoconstriction). The remaining 104 patients (58\%) had a normal renal $\mathrm{RI}$.

Laboratory evidence of kidney dysfunction developed in $48(27 \%)$ patients during follow-up. Forty-two of these 48 patients had an elevated renal RI. In $55 \%(42 / 76)$ of patients with elevated RIs, kidney dysfunction developed, whereas subsequent kidney dysfunction developed in only $6 \%(6 / 104)$ of those with normal RIs ( $p<0.00005)$. Cox regression analysis for kidney dysfunction with the use of the individual parameters previously identified in this study found that RI $(\mathrm{p}<0.00005)$, bilirubin level $(\mathrm{p}<0.0001)$, ascites $(\mathrm{p}<0.001)$ and age $(\mathrm{p}<0.05)$ were significant predictors of subsequent kidney dysfunction in this model. Serum albumin level, serum sodium level, mean arterial pressure, serum creatinine level, PT and encephalopathy were not significant in this analysis. The estimated survival function-type curves for kidney dysfunction based on RI values while controlling for covariants are shown in Figure 2.

Hepatorenal syndrome developed in 21 patients $(12 \%)$ of our study population. The mean follow-up time for all patients was 263 days, and the mean time for development of the hepatorenal syndrome was 63 days. In the patients in whom hepatorenal syndrome developed, the mean initial RI was $0.77 \pm 0.05$, which was significantly higher than that in patients in whom the hepatorenal syndrome did not develop $(0.67 \pm 0.07)(p<0.01)$. Subsequent development of hepatorenal syndrome was
TABLE 2. Relationship of renal RI at presentation to development of hepatorenal syndrome in 180 nonazotemic patients with liver disease

\begin{tabular}{lccc}
\hline Status & Normal RI $(<0.70)$ & Elevated RI $(\geq 0.70)$ & Total \\
\hline No HRS & 103 & 56 & 159 \\
HRS & 1 & 20 & 21 \\
\hline
\end{tabular}

HRS, hepatorenal syndrome.

The association between RI and subsequent development of hepatorenal syndrome was statistically significant $(p<0.001)$.

significantly related to renal RI status as indicated by Table 2. Twenty of the 21 patients in whom hepatorenal syndrome subsequently developed had an elevated RI at presentation (Fig. 3). Hepatorenal syndrome developed in $26 \%(20 / 76)$ of patients with an elevated RI, whereas only $1 \%(1 / 104)$ of patients with a normal renal RI went on to have hepatorenal syndrome. Hepatorenal syndrome development was an ominous prognostic sign because 17 of these 21 patients died. For all 180 patients, survival to either death or liver transplantation for 4 mo from the date of renal Doppler examination was observed in $99 \%$ of patients with a normal renal RI but in only $75 \%$ of those with an elevated RI ( $p<0.01$ ).

Cox regression analysis was performed for the outcome of hepatorenal syndrome with the individual parameters assessed in this study. Renal RI was a highly significant predictor of subsequent hepatorenal syndrome development $(p<0.00005)$. Serum bilirubin level $(\mathrm{p}<0.05)$ and PT $(\mathrm{p}<0.05)$ were also significant predictors of hepatorenal syndrome in this model. Serum albumin and sodium levels, ascites, encephalopathy, mean arterial pressure, serum creatinine concentration and age were not significantly related to subsequent hepatorenal syndrome development.

The mean RI value for the patients in whom the hepatorenal syndrome developed $(0.77 \pm 0.05)$ was significantly higher than for the 27 patients in whom kidney disease not classified as hepatorenal syndrome developed (mean RI, $0.72 \pm 0.04)(p<0.05)$ and the 132 patients who had no kidney dysfunction (mean RI, $0.65 \pm 0.08)(p<0.01)$. Patients with kidney disease but without the hepatorenal syndrome had a significantly higher initial renal $\mathrm{RI}$ value than patients without subsequent kidney dysfunction $(p<0.05)$.

Cause of liver disease was not a significant factor affecting the RI value. For each disease group the mean RI was between 0.66 and 0.70 with no statistically significant differences between diagnostic categories. Elevated RIs were seen more commonly in decompensated patients. An elevated RI was observed in 4 of 32 (12\%) Child-Pugh class A patients, 35 of 89 (39\%) class B patients and 37 of 59 (63\%) class C patients.

Although an elevated RI was more commonly seen with more advanced liver disease as shown by Child-Pugh class, the RI did not merely reflect the degree or decompensation of liver function (Table 3). This point is well illustrated by data for the subgroup of Child-Pugh class $\mathrm{C}$ patients highlighted in Table 4. 


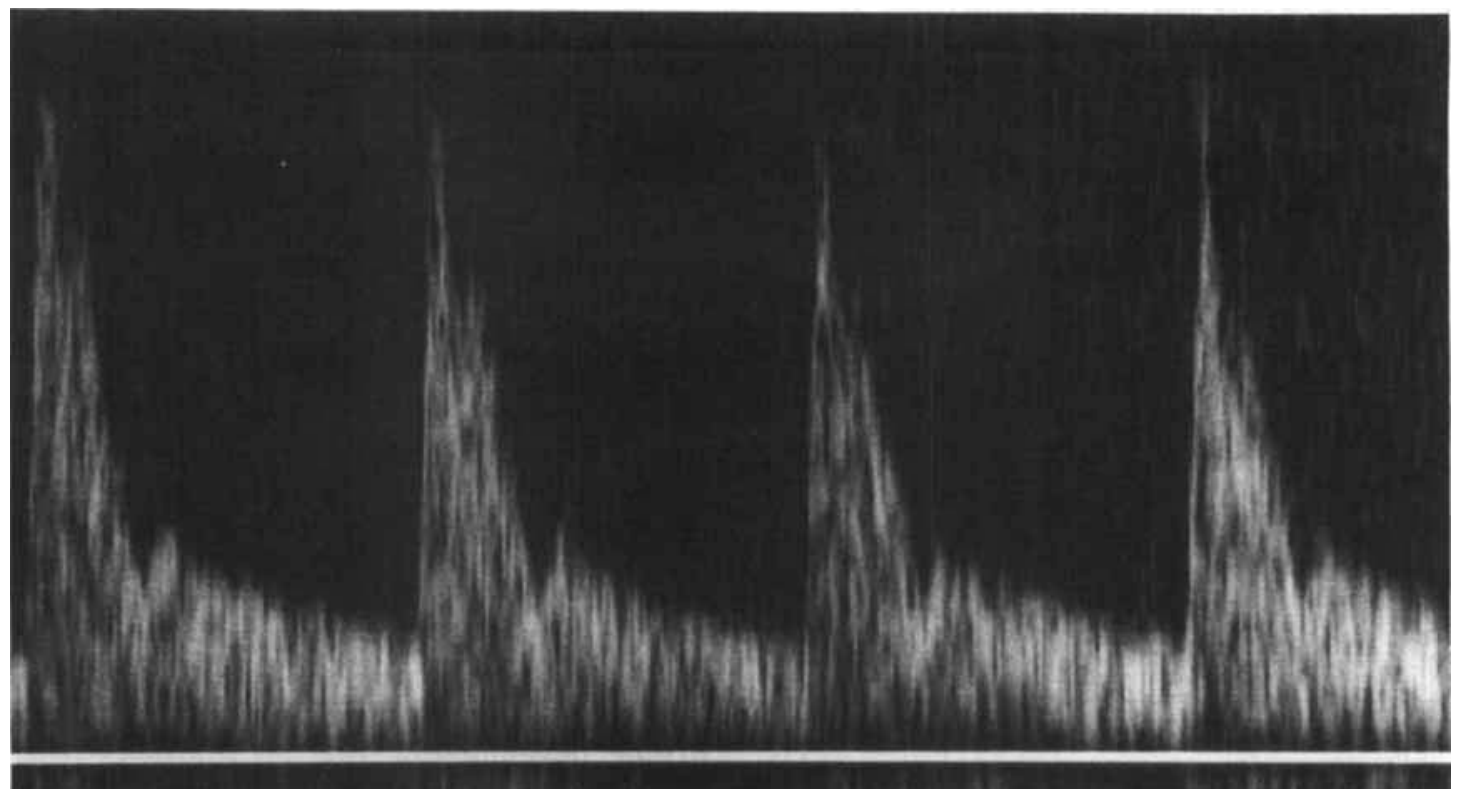

FIG. 3. Abnormal renal Doppler waveform (RI, 0.84) in a patient who was clinically nonazotemic (creatinine level, $1.0 \mathrm{mg} / \mathrm{dl}$ ). Hepatorenal syndrome developed in this patient 8 wk later.

TABLE 3. Relationship between severity of liver disease, RI and kidney outcome in 180 patients

\begin{tabular}{|c|c|c|c|c|c|c|}
\hline \multirow[b]{2}{*}{ Child-Pugh class } & \multirow[b]{2}{*}{$\mathbf{R I}$} & \multirow[b]{2}{*}{ No. of patients } & \multicolumn{2}{|c|}{ HRS } & \multicolumn{2}{|c|}{ RD } \\
\hline & & & $\overline{\mathbf{N}}$ & $\%^{a}$ & $\overline{\mathbf{n}}$ & $\%^{a}$ \\
\hline A & Normal & 28 & 0 & & 0 & \\
\hline $\mathrm{B}$ & Normal & 54 & 1 & 2 & 3 & 6 \\
\hline B & Elevated & 35 & 4 & 11 & 13 & 37 \\
\hline C & Normal & 22 & 0 & & 3 & 14 \\
\hline
\end{tabular}

$H R S$, Hepatorenal syndrome; $R D$, laboratory evidence for kidney dysfunction (see text).

${ }^{a}$ Percentage of patients in the subclassification in whom the finding developed.

Despite similar Child-Pugh scores, the subsequent kidney outcome was considerably worse in those patients with an elevated RI, in whom kidney dysfunction and hepatorenal syndrome occurred in $78 \%$ and $43 \%$, respectively, compared with $14 \%$ and $0 \%$, respectively, in those Child-Pugh class $\mathrm{C}$ patients in whom the renal RI was normal.

Although the patients with an initially elevated creatinine level were excluded from the primary focus of this study, data analysis revealed a mean RI of 0.77 in these 67 patients with all but 11 patients having an elevated RI. The hepatorenal syndrome did not develop in any of the 11 patients with a normal RI but an elevated creatinine level, and the creatinine level subsequently decreased to normal in 9 of these 11 .

\section{DISCUSSION}

The hepatorenal syndrome is a well-recognized complication of liver failure that often appears to develop acutely in previously nonazotemic patients (1). The earliest stages of this apparently functional form of kidney failure often go unrecognized because creatinine elevation is a late feature of the hepatorenal syndrome spectrum (1-3). Intense intrarenal vasoconstriction is an early hallmark of this functional kidney failure, although the precise causes are poorly defined and clinical assessment of the vasoconstriction has up to now been difficult (8-11). We have applied renal duplex Doppler ultrasonography, a widely available noninvasive modality, to the identification of this early kidney vasoconstriction in nonazotemic patients with established liver disease. Through use of a simply measured and easily obtained parameter, the RI, patients with probable kidney vasoconstriction can be quickly identified. We hypothesized that these patients with an elevated RI (presumably reflecting intrarenal vasoconstriction) would be at greater risk for development of overt hepatorenal syndrome. We have found renal RI to be a useful new noninvasive predictor of subsequent kidney status in nonazotemic patients with liver disease.

We analyzed our data for a poor kidney outcome, which we termed kidney dysfunction (doubling of initial creatinine level to at least $1.5 \mathrm{mg} / \mathrm{dl}$ ). This outcome variable encompasses the patients with hepatorenal 
TABLE 4. Comparison of parameters and outcomes in 59 Child-Pugh class $C$ patients categorized by renal RI

\begin{tabular}{lccr}
\hline \multicolumn{1}{c}{ Parameters } & Normal RI & p Value & Elevated RI \\
No. of patients & 22 & & 37 \\
Albumin $(\mathrm{gm} / \mathrm{dl})^{a}$ & $2.6 \pm 0.4$ & NS & $2.7 \pm 0.5$ \\
Bilirubin $(\mathrm{mg} / \mathrm{dl})^{a, b}$ & $7.0 \pm 6.3$ & NS & $8.2 \pm 7.5$ \\
PT $(\mathrm{sec})^{a}$ & $15.6 \pm 1.6$ & NS & $16.4 \pm 2.1$ \\
Child-Pugh score $^{a}$ & $10.5 \pm 1.0$ & NS & $11.2 \pm 1.1$ \\
${\text { Creatinine }(\mathrm{mg} / \mathrm{dl})^{a, c}}_{\text {HRS }(\%)^{d}}$ & $0.9 \pm 0.3$ & NS & $1.0 \pm 0.2$ \\
RD $(\%)^{d}$ & 0 & $<0.001$ & $43(16 / 37)$ \\
\hline
\end{tabular}

$N S$, Not significant; $H R S$, hepatorenal syndrome; $R D$, laboratory evidence for renal dysfunction.

${ }^{a}$ Data expressed as mean \pm S.D.

${ }^{b}$ To convert bilirubin to micromoles per liter multiply by 17.1 .

cTo convert values for creatinine to micromoles per liter multiply by 88.4

${ }^{d}$ Numbers in parentheses are numbers of patients.

syndrome and adds patients in our series who had often significant kidney failure that did not meet formal criteria for hepatorenal syndrome during the time of study. Prior reports have identified an elevated creatinine level to be a very important risk factor for morbidity and mortality, both before and after liver transplantation $(19,21,22)$. In our study of patients with a normal serum creatinine level, kidney dysfunction developed in $27 \%$ (48/180), $42(88 \%)$ of whom had an elevated renal RI. In contrast, only $6 \%(6 / 104)$ of patients with a normal RI had kidney dysfunction. RI was a significant independent predictor of subsequent kidney dysfunction, on the basis of Cox regression analysis.

Hepatorenal syndrome developed in $12 \%(21 / 180)$ of our study group. An elevated renal RI was observed at a time when serum creatinine level was within normal limits in $95 \%(20 / 21)$ of patients in whom hepatorenal syndrome later developed. Hepatorenal syndrome developed in only $1 \%(1 / 104)$ of patients with a normal renal RI. Therefore, those patients with a normal RI, which presumably indicated the lack of pathologic vasoconstriction, were at very small risk for overt hepatorenal syndrome. Interestingly, the one patient with a normal renal RI in whom hepatorenal syndrome developed was observed again and found to have a markedly elevated RI $(0.84)$ at the time of clinical hepatorenal syndrome. The time interval between initial Doppler study and hepatorenal syndrome in this patient was 220 days, and it is possible that this patient also had an elevated RI long before presenting with overt hepatorenal syndrome.

For the prediction of hepatorenal syndrome we compared RI with the components of the Child-Pugh classification (20), serum creatinine level, serum sodium level, mean arterial pressure and patient age. We found $\mathrm{RI}$ to be a significant independent prognostic variable of subsequent hepatorenal syndrome by Cox proportional hazards regression analysis.

Although almost all patients in whom hepatorenal syndrome developed and most in whom kidney dysfunction developed had an elevated renal RI, not all patients with an elevated RI had a poor kidney outcome.
Prior clinical reports suggest that functional kidney failure and vasoconstriction are much more prevalent than overt hepatorenal syndrome $(2,8,23-25)$. It is therefore possible that an additional insult such as sepsis, bleeding, excessive diuresis or nephrotoxic drug administration is necessary to hasten the development of clinical hepatorenal syndrome among the group of at-risk patients (those already with renal vasoconstriction reflected by an elevated $\mathrm{RI}$ ).

The relationship between severity of liver disease, renal RI and clinical kidney outcome is illustrated in Table 3. Although the severity of liver disease is related to observed RI, with RI elevation much more common in class $\mathrm{C}$ than class A patients, it would be a mistake to consider the RI as merely providing repetitive information to traditional parameters. This point is highlighted by data in Table 4 showing RI to be a useful predictor of kidney outcome among the patients with the most severe liver disease (Child class $\mathrm{C}$ ). Hepatorenal syndrome did not develop in any Child-Pugh class $\mathrm{C}$ patient with a normal RI. Furthermore, patients with less severe liver disease (class B) and an elevated RI had more frequent poor kidney outcomes than more decompensated class $\mathrm{C}$ patients with a normal RI (Table 3 ). Combining the clinical and RI data allowed identification of a subgroup of patients (class $\mathrm{C}$ and elevated RI) at highest risk for kidney dysfunction and hepatorenal syndrome.

The cause of liver disease was not a significant predictor of observed renal RI. Our data would suggest that the early stage of intrarenal vasoconstriction is seen broadly across most causes of liver disease because we observed almost identical mean $\mathrm{RI}$ values for each major liver disease category.

Duplex Doppler ultrasonography is widely available in the medical community. The examination is quick and noninvasive. In our study the renal Doppler ultrasound study was technically successful in all patients; no patient had to be excluded because of our inability to obtain a RI measurement. At our institution almost all patients in whom liver transplantation is considered undergo a right upper quadrant ultrasound examination with duplex Doppler study of hepatic vasculature. 
Therefore, the addition of a renal Doppler study would take only a few minutes in these patients already in the sonography suite.

Several potential limitations of our study are important to consider. First, a number of nonazotemic patients $(n=60)$ initially examined with renal Doppler ultrasonography were not included in this study because no appropriate follow-up data could be obtained. Although this exclusion is a possible source of bias, the patients so excluded were not selected on the basis of Doppler findings, and we believe our resulting study population to be representative of the patient population being seen in the liver clinics of our institution. These 60 patients had a similar percentage of elevated RIs (40\%) as compared with our study population $(42 \%)$ and a similar severity of liver disease (mean Child-Pugh score, $8.7 \pm 1.8$ ) as compared with our study population (mean score, $8.5 \pm 2.1$ ). In our study, we used serum creatinine concentration as an indicator of the patients' initial kidney status. More sophisticated types of kidney evaluation such as glomerular filtration rate were not used. Other kidney pathologic states besides hepatorenal syndrome and functional kidney failure are capable of elevating the RI (12-16). Kidney obstruction, acute tubular necrosis, renal vein thrombosis and the hemolyticuremic syndrome all can cause RI elevation (12-16).

However, these patients in general have clinically recognized kidney dysfunction accompanying the elevated renal RI, and neither the clinical syndromes nor the kidney dysfunction were recognized during evaluation of the clinically nonazotemic patients in our study. Last, this study was not designed to assess changes in renal RI over time. Longitudinal data of this type were beyond the scope of this initial investigation but may be valuable as predictors of impending hepatorenal syndrome.

In conclusion, we have demonstrated that renal duplex Doppler ultrasound examination noninvasively identifies a subgroup of nonazotemic patients with established liver disease at higher risk for subsequent kidney dysfunction and hepatorenal syndrome by presumably detecting renal vasoconstriction. An abnormal renal RI predicts an increased chance for development of the hepatorenal syndrome and kidney dysfunction independent of liver disease severity and is a better predictor than the individual parameters of the Child-Pugh classification. When an elevated RI is obtained, a formal evaluation for hepatorenal syndrome including fluid challenge should be considered. A normal renal Doppler study would indicate that hepatorenal syndrome is very unlikely. Doppler information will be useful for prognosis and in the management of liver disease in patients whenever they require paracentesis, diuretic therapy, potentially nephrotoxic medications or radiographic contrast examination. In the future, renal RI monitoring may prove to be valuable in assessing therapies designed to maintain normal renal vascular tone $(26,27)$ and in recognizing patients in whom early liver transplantation may be desirable.
Acknowledgments: We thank Elaine Grech for the preparation of this manuscript, Suzanne Maloney for the performance and measurement of the Doppler studies and Kathy Welch for statistical consultation.

\section{REFERENCES}

1. Epstein M. Hepatorenal syndrome. In: Epstein M, ed. The kidney in liver disease. 3rd ed. Baltimore: Williams \& Wilkins, 1988: 89-118.

2. Gentilini P, Laffi G. Renal functional impairment and sodium retention in liver cirrhosis. Digestion 1989;43:1-32.

3. Schelling JR, Linas SL. Hepatorenal syndrome. Semin Nephrol 1990;10:565-570.

4. Koppel MH, Coburn JW, Mims MM, Goldstein H, Boyle JD, Rubini ME. Transplantation of cadaveric kidneys from patients with hepatorenal syndrome: evidence for the functional nature of renal failure in advanced liver disease. N Engl J Med 1969;280:1367. 1371.

5. Gonwa TA, Poplawski S, Paulsen W, Brajtbord D, Goldstein R, Husberg B, Klintmalm GB. Pathogenesis and outcome of hepatorenal syndrome in patients undergoing orthotopic liver transplant. Transplantation 1989;47:395-397.

6. Iwatsuki S, Papovtzer MM, Corman JL, et al. Recovery from "hepatorenal syndrome" after orthotopic liver transplantation. N Engl J Med 1973;289:1155-1160.

7. Papadakis MA, Arieff AI. Unpredictability of clinical evaluation of renal function in cirrhosis. Am J Med 1987;82:945-952.

8. Gentilini P, Laffi G, Buzzelli G, Stefani P, Scarpelli P, Paladini S, Smorlesi C, et al. Functional renal alterations in chronic liver diseases. Digestion 1980;20:73-78.

9. Epstein M, Berk DP, Hollenberg NK, Adams DF, Chalmero TC, Abrams HL, Merrill JP. Renal failure in the patient with cirrhosis: the role of active vasoconstriction. Am J Med 1970;49:175-185.

10. Epstein M. The hepatorenal syndrome. Hosp Pract 1989;24:65-76

11. Moore K, Wendon J, Frazer M, Karani J, Williams R, Badr K. Plasma endothelin immunoreactivity in liver disease and the hepatorenal syndrome. N Engl J Med 1992;327:1774-1778.

12. Platt JF. Duplex Doppler evaluation of native kidney dysfunction: obstructive and nonobstructive disease. A.JR Am J Roentgenol 1992;158:1035-1042.

13. Platt JF, Rubin JM, Ellis JH. Distinction between obstructive and nonobstructive pyelocaliectasis with duplex Doppler sonography. AJR Am J Roentgenol 1989;153:997-1000.

14. Platt JF, Rubin JM, Ellis JH. Acute renal failure: possible role of duplex Doppler US in distinction between acute prerenal failure and acute tubular necrosis. Radiology 1991;179:419-423.

15. Parvey HR, Eisenberg RL. Image-directed Doppler sonography of the intrarenal arteries in acute renal vein thrombosis. JCU J Clin Ultrasound 1990;18:512-516.

16. Patriquin HB, O'Regan S, Robitaille P, Paltiel H. Hemolyticuremic syndrome: intrarenal arterial Doppler patterns as a useful guide to therapy. Radiology $1989 ; 172: 625-628$.

17. Platt JF, Marn CS, Baliga PK, Ellis JH, Rubin JM, Merion RM Renal dysfunction in hepatic disease: early identification with renal duplex Doppler US in patients who undergo liver transplantation. Radiology 1992;183:801-806.

18. Colli A, Cocciolo M, Riva C, Martinez E. Abnormal renovascular impedance in patients with hepatic cirrhosis: detection with duplex US. Radiology 1993;187:561-563.

19. Cuervas-Mons V, Millan I, Gavaler JS, Starzl TE, Van Thiel DH Prognostic value of preoperatively obtained clinical and laboratory data in predicting survival following orthotopic liver transplantation. HePATOLOGY 1986;6:922-927.

20. McCurry KR, Baliga $P$, Merion RM, Ham JM, Lucey MR, Beresford TP, Turcotte JG, et al. Resource utilization and outcome of liver transplantation for alcoholic cirrhosis. Arch Surg 1992;127:772-777.

21. Rimola A, Gavaler JS, Schade RR, El-Lankany S, Starzl TE, Van Thiel DH. Effects of renal impairment on liver transplantation. Gastroenterology 1987;93:148-156. 
22. Gonwa TA, Poplawski S, Paulsen W, Brajtbord D, Goldstein R, Husberg B, Klintmalm G. Hepatorenal syndrome and orthotopic liver transplantation. Transplant Proc 1989;21:2419-2420.

23. Fernandez-Seara J, Prieto J, Quiroga J, Zozaya JM, Cobos MA Rodriguez-Eire JL, Garcia-Plaza A, et al. Systemic and regional hemodynamics in patients with liver cirrhosis and ascites with and without functional renal failure. Gastroenterology 1989;97:13041312.

24. Tristani FE, Cohn JN. Systemic and renal hemodynamics in oliguric hepatic failure: effect of volume expansion. J Clin Invest 1967;46:1894-1906.
25. Ring-Larsen H. Hepatic nephropathy, related to hemodynamics Liver 1983;3:265-289.

26. Lenz K, Hortnagl H, Druml W, Reither H, Schmid R, Schneeweiss B, Laggner A, et al. Ornipressin in the treatment of functional renal failure in decompensated liver cirrhosis. Gastroenterology 1991;101:1060-1067.

27. Arroyo V, Gines P. Prostaglandins and the treatment of hepatorenal syndrome in cirrhosis. J Hepatol 1990;11:142-144. 